\title{
NOUVELLE
}

\section{Un rôle pour la protéine embryonnaire Lin-28 dans la différenciation myogénique}

Anna Polesskaya, Annick Harel-Bellan
CNRS, FRE 2944, Institut André Lwoff, F-94801 Villejuif, France.

Université Paris-Sud, F-94801Villejuif, France. apoles@vjf.cnrs.fr
> Lin-28 est une protéine se liant à I'ARN, et caractérisée par une combinaison unique de motifs CSD (cold shock domains) et ZFM (zinc finger motifs). Très conservée entre les espèces, Lin-28 est indispensable pour le développement embryonnaire précoce, pendant lequel son expression est contrôlée par plusieurs facteurs protéiques et par des microARN (miRs) [1, 2]. Dans la majorité des tissus adultes, l'expression de Lin-28 s'éteint, à l'exception toutefois des muscles squelettique et cardiaque [3]. Malgré l'importance physiologique de Lin-28 pour le développement, aucune fonction n'a été attribuée à cette protéine jusqu'à ce jour.

\section{Lin-28 interagit avec le complexe d'initiation de la traduction} Nous avons étudié le rôle de Lin-28 dans le muscle squelettique [4]. Dans les myoblastes primaires de souris, Lin-28 est fortement induite au cours de la différenciation terminale, aboutissant à la formation des myotubes multinucléés, pendant laquelle elle est absolument indispensable, comme nous l'avons démontré par la technique d'ARN interférence (ARNi). En utilisant une approche protéomique, nous avons montré que Lin-28 interagissait avec le complexe d'initiation de la traduction. Pendant la différenciation musculaire, Lin-28 est associée aux polysomes, comme nous l'avons montré en fractionnant des extraits de myoblastes sur un gradient de sucrose. Dans les myoblastes en phase de prolifération ou en cours de différenciation, Lin-28 est distribuée de façon diffuse dans le cytoplasme, où elle co-localise avec les complexes d'initiation de la traduction. Lorsque les cellules sont soumises à un stress oxydatif, Lin-28 est séquestrée dans les «granules de stress», des structures cellulaires temporaires contenant les complexes abortifs de la traduction, ainsi que les facteurs régulateurs de la traduction [5] (Figure I). Finalement, nous avons trouvé que pendant la myogenèse, Lin-28 augmente l'efficacité de la traduction de certains de ses ARNm cibles.

\section{Lin-28 active la traduction de IGF-2}

$\varepsilon$ n effet, nos résultats suggèrent qu'une des fonctions principales de Lin-28 dans le muscle est l'activation de la traduction de l'ARNm codant pour IGF-2 (insulin-like growth factor-2), un facteur autocrine essentiel pour la myogenèse embryonnaire et adulte, comme pour le développement embryonnaire en général. L'induction de Lin-28 pendant la différenciation myogénique induit le recrutement de l'ARNm de l'IGF-2 dans les polysomes actifs (Figure 2). Quand l'expression de Lin-28 dans les myoblastes primaires est supprimée par les siARN spécifiques, I'ARNm de I'IGF-2 reste associé aux monosomes, et le taux de synthèse de la protéine IGF-2 s'effondre. La différenciation terminale des myoblastes est extrêmement sensible à la présence et à la concentration exacte d'IGF-2, ce qui nous incite à conclure que la régulation de la myogenèse par Lin-28 passe en grande partie par l'intermédiaire de cette cytokine IGF-2. Cette hypothèse est confirmée par la totale inhibition de l'effet positif de Lin-28 sur la différenciation myogénique en présence d'anticorps anti-IGF-2.

L'expression d'IGF-2 est régulée à plusieurs niveaux [6]. Notamment, l'efficacité de la transcription du gène IGF-2 échappe au contrôle par Lin-28. À ce propos, il est intéressant de noter que l'induction de la protéine IGF-2 dans les myoblastes primaires et dans

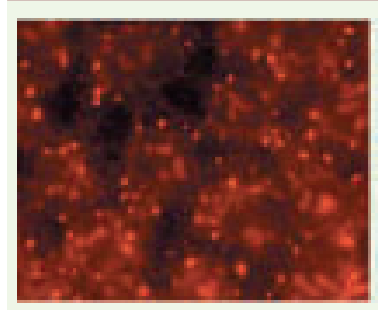

Lin-28

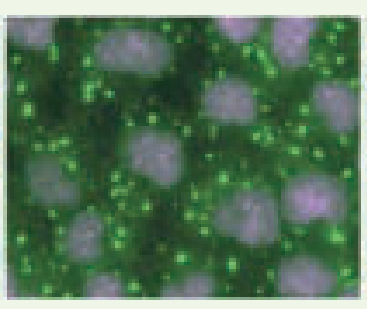

TIA-1/DAPI

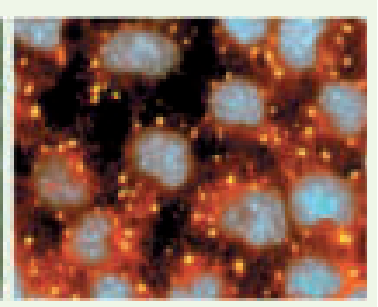

Co-localisation

Figure 1. Localisation de Lin-28 dans les myoblastes primaires. Des myoblastes primaires de souris en phase de prolifération ont été traités avec $500 \mathrm{nM}$ d'arsenate de sodium pendant 30 minutes. Les granules de stress, complexes abortifs de la traduction, sont reconnus par le marqueur classique TIAl (vert). Lin-28 (rouge) co-localise avec TIA-l dans les granules de stress. Les noyaux sont colorés avec le DAPI. 
le muscle in vivo ne requiert qu'une activation transcriptionnelle faible et dépend essentiellement de la régulation post-transcriptionnelle, notamment par Lin-28. À l'inverse, les lignées établies de myoblastes ou les cellules tumorales de rhabdomyosarcome n'expriment plus Lin-28, mais sont capables d'activer la transcription de l'IGF-2 de manière extrêmement importante. Cette observation suggère que la régulation traductionnelle par Lin-28 (et peut-être par d'autres protéines se liant à l'ARNm) caractérise plutôt les cellules et tissus sains, tandis que dans les tissus tumoraux, l'expression de l'IGF-2 est assurée par l'augmentation de l'activité transcriptionnelle. Cependant, une surexpression importante d'un homologue de Lin-28 a récemment été décrite dans un carcinome hépatocellulaire humain, suggérant que des changements dans cette voie de régulation peuvent être impliqués dans certains cas de transformation maligne [7].

Lin-28 est très fortement exprimée dans les cellules souches embryonnaires (cellules ES) et pendant le développement embryonnaire précoce, et il est probable que le mécanisme d'activation traductionnelle par Lin-28 que nous avons décrit est aussi utilisé à cette étape du développement où la synthèse des protéines est très active. Le rôle de Lin-28 dans les cellules $E S$ reste cependant à démontrer, et rien n'est connu des ARNm cibles régulés par ce facteur au cours de l'embryogenèse. Notre travail a démontré que Lin-28 contrôle l'expression de la cytokine IGF-2 pendant la myogenèse, proposant une importante fonction dans la régulation traductionnelle pour cette petite protéine se liant à l'ARNm. D’autres études seront nécessaires pour identifier toutes les cibles régulées par Lin-28, et comprendre pourquoi cette protéine est indispensable pour le développement en général, et pour la maintenance de certains tissus chez l'adulte. $\diamond$

A novel role for an embryonic regulatory protein Lin-28 in adult skeletal muscle differentiation

\section{RÉFÉRENCES}

1. Moss \&G, Lee RC, Ambros V. The cold shock domain protein LIN-28 controls developmental timing in C. elegans and is regulated by the lin- 4 RNA. Cell 1997; $88: 637-46$

2. Sempere LF, Freemantle S, Pitha-Rowe I, et al. Expression profiling of mammalian microRNAs uncovers a subset of brain-expressed microRNAs with possible roles in murine and human neurona differentiation. Genome Biol 2004 ; 5 : R13.

3. Yang D, Moss $\varepsilon G$. Temporally regulated expression of Lin-28 in diverse tissues of the developing mouse. Gene Expr Patterns 2003 ; 3 : 719-26.

4. Polesskaya A, Cuvellier S, Naguibneva I, et al. Lin-28 binds IGF-2 mRNA and participates in skeletal myogenesis by increasing translation efficiency. Genes Dev 2007 ; 21 : 1125-38.

5. Kedersha N, Anderson P. Stress granules: sites of mRNA triage that regulate mRNA stability and translatability. Biochem Soc Trans 2002; 30 : 963-9. 6. Florini JR, Ewton DZ, Coolican SA. Growth hormone and the insulin-like growth factor system in myogenesis. Endocrinol Rev 1996; 17 : 481-517.

7. Guo $\mathrm{Y}$, Chen $\mathrm{Y}$, Ito $\mathrm{H}$, et al. Identification and characterization of lin-28 homolog B (LIN28B) in human hepatocellular carcinoma. Gene 2006; $384: 51-61$.

ILLUSTRATIONS DES ARTICLES (VIGNETIES): p. 819: cellules dendritiques de la muqueuse buccale (๔ photo Dominique Kaiserlian) -p. 826 : rétine (@ photo Omar Benzakour) - p. 834 : chromosomes marqués par un colorant fluorescent (photo Philippe Metezeau (C) Photothèque Inserm) - page 840 : coupe sagittale de cerveau humain (photoStéphane Lehéricy - (C) Photothèque Inserm) - p. 845 : cellules nerveuses (photo Pascal Dournaud - (C) Photothèque Inserm) - page 850 : structures cytoplasmiques liées à la dégradation $\operatorname{des}$ ARNm (@ photo Nicolas Cougot) - page 857 : monocyte (photo Dimitri Dantchev - (c) Photothèque Inserm) - page 862 : cellules dendritiques humaines, marqueur lysosomal Lamp-1 (vert) et complexes CMH-I (rouge) (@ photo Peter van Endert) - page 868: neurones de l'encéphale (photo Jean-Patrick Guéritaud - (C) Photothèque Inserm) - page 875 : séquences d’ADN (photo Bertrand Jordan - (c) Photothèque Inserm) - page 877 : vue tranversale d'ADN (photo Jean-Louis Martin - (c) Photothèque Inserm).

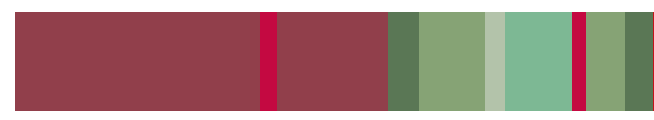

